Article

\title{
On a Neutral Itô and Arbitrary (Fractional) Orders Stochastic Differential Equation with Nonlocal Condition
}

\author{
Ahmed M. A. El-Sayed * and Hoda A. Fouad * \\ Faculty of Science, Alexandria University, Alexandria 21568, Egypt \\ * Correspondence: amasayed@alexu.edu.eg (A.M.A.E.-S.); hoda.fouad@alexu.edu.eg (H.A.F.)
}

check for updates

Citation: El-Sayed, A.M.A.; Fouad, H.A. On a Neutral Itô and Arbitrary (Fractional) Orders Stochastic Differential Equation with Nonlocal Condition. Fractal Fract. 2021, 5, 201. https: / / doi.org/10.3390/

fractalfract5040201

Academic Editor: Omar Abu Arqub

Received: 1 September 2021

Accepted: 1 November 2021

Published: 5 November 2021

Publisher's Note: MDPI stays neutral with regard to jurisdictional claims in published maps and institutional affiliations.

Copyright: (c) 2021 by the authors. Licensee MDPI, Basel, Switzerland. This article is an open access article distributed under the terms and conditions of the Creative Commons Attribution (CC BY) license (https:// creativecommons.org/licenses/by/ $4.0 /)$.
Abstract: In this paper, we are concerned with the combinations of the stochastic Ito-differential and the arbitrary (fractional) orders derivatives in a neutral differential equation with a stochastic, nonlinear, nonlocal integral condition. The existence of solutions will be proved. The sufficient conditions for the uniqueness of the solution will be given. The continuous dependence of the unique solution will be studied.

Keywords: stochastic processes; stochastic differential equation; Itô-differential; fractional differential equations; coupled system; nonlocal stochastic integral conditions

MSC: 34A12; 34A34; 34D20; 34K40; 60H10

\section{Introduction}

The existence and uniqueness of solutions to stochastic differential equations driven by the Winner Processes have been studied by many authors (see [1-5]).

In addition, the stochastic differential equations with nonlocal conditions and of fractional orders have been studied by some authors (see, for example, [6-8] and references therein).

The results are important since they cover nonlocal generalizations of differential SDEs, and more applications are arising in fields such as heat conduction, electromagnetic theory and dynamical systems and in materials with memory (see, e.g., [9-17]), optimal fractional problems and numerical models (see, e.g., [18-21]).

P. Balasubramaniam et al. [22] obtained sufficient conditions for the existence of a mild solution of the considered system by using analytic resolvent operators, the uniform continuity of the resolvent and Schauder fixed point theorem.

Here we study the existence of solutions of an Itô and arbitrary (fractional) orders stochastic nonlinear differential equation with nonlocal integral conditions containing the involved Caputo fractional order derivative. We combined two different senses of derivatives and stated the conditions for the existence of at least one solution.

Let $(W(t)), t \geq 0$ be a standard Brownian motion on a complete probability space $(\Omega, \digamma, \mu)$, where $\Omega$ is a sample space, $\digamma$ is a $\sigma$-algebra and $\mu$ is a probability measure.

Let $x(t ; \omega)=x(t), t \in[0, T], \omega \in \Omega$ be a second order stochastic process, i.e., $E\left(x^{2}(t)\right)<+\infty, t \in[0, T]$.

Let $C=C\left([0, T], L_{2}(\Omega)\right)$ be the space of all second order mean square (m.s) continuous stochastic processes on $[0, T]$. The norm of $x \in C\left([0, T], L_{2}(\Omega)\right)$ is given by

$$
\|x\|_{C}=\sup _{t \in[0, T]}\|x(t)\|_{2}, \quad \quad\|x(t)\|_{2}=\sqrt{E\left(x^{2}(t)\right)} .
$$

Let $\alpha, \beta \in(0,1]$ be such that $\alpha \geq \beta$ and $I=[0, T]$. In this paper we study the existence of solutions $x \in C\left(I, L_{2}(\Omega)\right)$ of the It $\hat{o}$ - arbitrary (fractional) orders stochastic nonlinear differential equation

$$
d\left(\frac{d x(t)}{d t}-g(t, x(\phi(t)))=f\left(t, D^{\alpha} x(t)\right)\right) d W(t), \quad t \in I
$$


subject to

$$
\begin{aligned}
\left.\frac{d x}{d t}\right|_{t=0} & =g(0, x(\phi(0))) \\
x(0) & +\int_{0}^{\tau} h\left(s, D^{\beta} x(s)\right) d W(s)=x_{0}
\end{aligned}
$$

where $x_{0}$ is a second-order random variable.

The existence of solutions $x \in C\left(I, L_{2}(\Omega)\right)$ of the problem (1) and (2) is proved. The sufficient conditions for the uniqueness of the solution will be given. The continuous dependence of the solution on the random variable $x_{0}$ and the random function $h$ will be studied. by [23].

The definitions of arbitrary (fractional) integral and derivatives have been studied

Definition 1. Let $x \in C\left(I, L_{2}(\Omega)\right)$ and $\alpha, \beta \in(0,1]$. The stochastic fractional order integral $I^{\beta} x(t)$ is defined by

$$
I^{\beta} x(t)=\int_{0}^{t} \frac{(t-s)^{\beta-1}}{\Gamma(\beta)} x(s) d s,
$$

and the stochastic fractional order derivative is defined by

$$
D^{\alpha} x(t)=I^{1-\alpha} \frac{d x}{d t} .
$$

For the properties to stochastic fractional calculus, see [23].

Consider the following assumptions

(B1) $\phi:[0, T] \rightarrow[0, T]$ is a continuous function such that $\phi(t) \leq t$.

(B2) $f: I \times L_{2}(\Omega) \rightarrow L_{2}(\Omega)$ is measurable in $t \in I$ for all $x \in L_{2}(\Omega)$ and continuous in $x \in L_{2}(\Omega)$ for all $t \in I$ and there exists a bounded measurable function $k: I \rightarrow R$ and a positive constant $b$ such that

$$
\|f(t, x)\|_{2} \leq|k(t)|+b\|x(t)\|_{2} .
$$

(B3) $h: I \times L_{2}(\Omega) \rightarrow L_{2}(\Omega)$ is measurable in $t \in I$ for all $x \in L_{2}(\Omega)$ and continuous in $x \in L_{2}(\Omega)$ for all $t \in I$ and there exists a bounded measurable function $m: I \rightarrow R$ and a positive constant $c$ such that

$$
\|h(t, x)\|_{2} \leq|m(t)|+c\|x(t)\|_{2} .
$$

(B4) $g: I \times L_{2}(\Omega) \rightarrow L_{2}(\Omega)$ is measurable in $t \in I$ for all $x \in L_{2}(\Omega)$ and continuous in $x \in L_{2}(\Omega)$ for all $t \in I$ and there exists a bounded measurable function $l: I \rightarrow R$ and a positive constant $q$ such that

$$
\|g(t, x)\|_{2} \leq|l(t)|+q\|x(t)\|_{2} .
$$

(B5) $K=\sup _{t \in I}|k(t)|, M=\sup _{t \in I}|m(t)|, L=\sup _{t \in I}|l(t)|$.

$$
\frac{b T^{\frac{3}{2}-\alpha}}{\Gamma(2-\alpha)}+\frac{q T^{1-\alpha}}{\Gamma(2-\alpha)}+\frac{c T^{\alpha-\beta+\frac{1}{2}}}{\Gamma(1+\alpha-\beta)}+\frac{T^{\alpha}}{\Gamma(1+\alpha)} \leq 1 .
$$

\section{Integral Representations of the Solution}

Integrating Equation (1) we obtain ([24]),

$$
\left.\frac{d x(t)}{d t}=g(t, x(\phi(t)))+\int_{0}^{t} f\left(t, D^{\alpha} x(t)\right)\right) d W(t),
$$


then operating by $I^{1-\alpha}$, we obtain

$$
\left.D^{\alpha} x(t)\right)=I^{1-\alpha} \frac{d x}{d t}=I^{1-\alpha} g(t, x(\phi(t)))+I^{1-\alpha} \int_{0}^{t} f\left(s, D^{\alpha} x(s)\right) d W(s) .
$$

Let

$$
\left.y(t)=D^{\alpha} x(t)\right)
$$

then we deduce that

$$
\begin{aligned}
y(t) & =I^{1-\alpha} g(t, x(\phi(t)))+I^{1-\alpha} \int_{0}^{t} f(s, y(s)) d W(s) \\
& =I^{1-\alpha} g(t, x(\phi(t)))+\int_{0}^{t} \frac{(t-s)^{-\alpha}}{\Gamma(1-\alpha)} \int_{0}^{s} f(\theta, y(\theta)) d W(\theta) d s \\
& =I^{1-\alpha} g(t, x(\phi(t)))+\int_{0}^{t} \frac{(t-s)^{-\alpha}}{\Gamma(1-\alpha)} \int_{0}^{s}\left(f(\theta, y(\theta)) \cdot \frac{d W}{d \theta}\right) d \theta \cdot d s \\
& =I^{1-\alpha} g(t, x(\phi(t)))+\int_{0}^{t} \frac{(t-s)^{1-\alpha}}{\Gamma(2-\alpha)} f(s, y(s)) d W(s) .
\end{aligned}
$$

Let $\alpha \geq \beta$, then $D^{\beta} x(t)=I^{\alpha-\beta} y(t)$. Now, integrating (6) we can obtain

$$
x(t)=x_{0}-\int_{0}^{\tau} h\left(s, I^{\alpha-\beta} y(s)\right) d W(s)+I^{\alpha} y(t) .
$$

Then the following lemma is proved.

Lemma 1. The integral representations corresponding to the solution of the nonlocal problem (1) and (2) is given by

$$
\begin{aligned}
& x(t)=x_{0}-\int_{0}^{\tau} h\left(s, I^{\alpha-\beta} y(s)\right) d W(s)+I^{\alpha} y(t), \\
& y(t)=I^{1-\alpha} g(t, x(\phi(t)))+\int_{0}^{t} \frac{(t-s)^{1-\alpha}}{\Gamma(2-\alpha)} f(s, y(s)) d W(s) .
\end{aligned}
$$

\section{Existence Theorem}

Let $\Lambda=C\left(I, L_{2}(\Omega)\right) \times C\left(I, L_{2}(\Omega)\right)$ be the class of all ordered pairs $(x, y), x, y \in C$ with the norm

$$
\|(x, y)\|_{\Lambda}=\|x\|_{C}+\|y\|_{C}=\sup _{t \in I}\|x(t)\|_{2}+\sup _{t \in I}\|y(t)\|_{2} .
$$

Define the mapping $F(x, y)=\left(F_{1} y, F_{2}(x, y)\right)$ where $F_{1} y, F_{2}(x, y)$ are given by the following stochastic integral equations

$$
\begin{aligned}
F_{1} y(t) & =x_{0}-\int_{0}^{\tau} h\left(s, I^{\alpha-\beta} y(s)\right) d W(s)+I^{\alpha} y(t), \\
F_{2}(x, y)(t) & =I^{1-\alpha} g(t, x(\phi(t)))+\int_{0}^{t} \frac{(t-s)^{1-\alpha}}{\Gamma(2-\alpha)} f(s, y(s)) d W(s) .
\end{aligned}
$$

Consider the set $Q$ such that

$$
Q=\left\{x, y \in C\left(I, L_{2}(\Omega)\right),(x, y) \in \Lambda:\|(x, y)\|_{\Lambda}=\left\{\|x(t)\|_{2}+\|y(t)\|_{2}\right\} \leq r\right\} .
$$

Now, we have the following existence theorem

Theorem 1. Let the assumptions (B1)-(B6) be satisfied, then there exists at least one solution $(x, y) \in \Lambda$ of the problem (9) and (10). 
Proof. Let $(x, y) \in Q$, then we have

$$
\begin{aligned}
\left\|F_{1} y(t)\right\|_{2} & \leq\left\|x_{0}\right\|_{2}+\left\|\int_{0}^{\eta} h\left(s, I^{\alpha-\beta} y(s)\right) d W(s)\right\|_{2}+\left\|I^{\alpha} y(t)\right\|_{2} \\
& \leq\left\|x_{0}\right\|_{2}+M \sqrt{T}+\left[\frac{c T^{\alpha-\beta+\frac{1}{2}}}{\Gamma(1+\alpha-\beta)}+\frac{T^{\alpha}}{\Gamma(1+\alpha)}\right]\|y\|_{C}
\end{aligned}
$$

and

$$
\begin{aligned}
\left\|F_{2}(x, y)(t)\right\|_{2} & \left.\leq \| I^{1-\alpha} g(t, x(\phi(t)))\right)\left\|_{2}+\right\| \int_{0}^{t} \frac{(t-s)^{1-\alpha}}{\Gamma(2-\alpha)} f(s, y(s)) d W(s) \|_{2} \\
& \leq \frac{T^{1-\alpha}}{\Gamma(2-\alpha)}\left[L+q\|x\|_{C}\right] \\
& +\sqrt{\int_{0}^{t}\left\|\frac{(t-s)^{1-\alpha}}{\Gamma(2-\alpha)} f(s, y(s))\right\|_{2}^{2} d s} \\
& \leq \frac{L T^{1-\alpha}}{\Gamma(2-\alpha)}+\frac{K T^{\frac{3}{2}-\alpha}}{\Gamma(2-\alpha)}+\frac{q T^{1-\alpha}\|x\|_{C}}{\Gamma(2-\alpha)}+\frac{b T^{\frac{3}{2}-\alpha}\|y\|_{C}}{\Gamma(2-\alpha)} .
\end{aligned}
$$

This implies that

$$
\begin{aligned}
\|F(x, y)\|_{\Lambda} & =\left\|\left(F_{1} y, F_{2}(x, y)\right)\right\|_{\Lambda} \\
& =\left\|F_{1} y\right\|_{C}+\left\|F_{2} x\right\|_{C} \\
& \leq\left\|x_{0}\right\|_{2}+M \sqrt{T}+\frac{L T^{1-\alpha}}{\Gamma(2-\alpha)}+\frac{K T^{\frac{3}{2}-\alpha}}{\Gamma(2-\alpha)} \\
& +\frac{q T^{1-\alpha}\|x\|_{C}}{\Gamma(2-\alpha)}+\left[\frac{b T^{\frac{3}{2}-\alpha}}{\Gamma(2-\alpha)}+\frac{c T^{\alpha-\beta+\frac{1}{2}}}{\Gamma(1+\alpha-\beta)}+\frac{T^{\alpha}}{\Gamma(1+\alpha)}\right]\|y\|_{C} \\
\leq & \left\|x_{0}\right\|_{2}+M \sqrt{T}+\frac{L T^{1-\alpha}}{\Gamma(2-\alpha)}+\frac{K T^{\frac{3}{2}-\alpha}}{\Gamma(2-\alpha)} \\
+ & {\left[\frac{q T^{1-\alpha}}{\Gamma(2-\alpha)}+\frac{b T^{\frac{3}{2}-\alpha}}{\Gamma(2-\alpha)}+\frac{c T^{\alpha-\beta+\frac{1}{2}}}{\Gamma(1+\alpha-\beta)}+\frac{T^{\alpha}}{\Gamma(1+\alpha)}\right]\left[\|x\|_{C}+\|y\|_{C}\right] } \\
\leq & \left\|x_{0}\right\|_{2}+M \sqrt{T}+\frac{L T^{1-\alpha}}{\Gamma(2-\alpha)}+\frac{K T^{\frac{3}{2}-\alpha}}{\Gamma(2-\alpha)} \\
+ & {\left[\frac{q T^{1-\alpha}}{\Gamma(2-\alpha)}+\frac{b T^{\frac{3}{2}-\alpha}}{\Gamma(2-\alpha)}+\frac{c T^{\alpha-\beta+\frac{1}{2}}}{\Gamma(1+\alpha-\beta)}+\frac{T^{\alpha}}{\Gamma(1+\alpha)}\right] r=r }
\end{aligned}
$$

where

$$
r=\frac{\left\|x_{0}\right\|_{2}+M \sqrt{T}+\frac{L T^{1-\alpha}}{\Gamma(2-\alpha)}+\frac{K T^{\frac{3}{2}-\alpha}}{\Gamma(2-\alpha)}}{1-\left[\frac{q T^{1-\alpha}}{\Gamma(2-\alpha)}+\frac{b T^{\frac{3}{2}-\alpha}}{\Gamma(2-\alpha)}+\frac{c T^{\alpha-\beta+\frac{1}{2}}}{\Gamma(1+\alpha-\beta)}+\frac{T^{\alpha}}{\Gamma(1+\alpha)}\right]},
$$

then the class $\{F(x, y)\}$ is uniformly bounded and $F(x, y): Q \rightarrow Q$.

Let $(x, y) \in Q, \quad t_{1}, t_{2} \in[0, T], \quad t_{1}<t_{2}$ such that $\left|t_{2}-t_{1}\right|<\delta$, then

$$
\begin{aligned}
F_{1} y\left(t_{2}\right)-F_{1} y\left(t_{1}\right) & =\int_{0}^{t_{2}} \frac{\left(t_{2}-s\right)^{\alpha-1}}{\Gamma(\alpha)} y(s) d s-\int_{0}^{t_{1}} \frac{\left(t_{1}-s\right)^{\alpha-1}}{\Gamma(\alpha)} y(s) d s \\
& =\frac{1}{\Gamma(\alpha)} \int_{0}^{t_{1}}\left[\left(t_{2}-s\right)^{\alpha-1}-\left(t_{1}-s\right)^{\alpha-1} y(s) d s+\frac{1}{\Gamma(\alpha)} \int_{t_{1}}^{t_{2}}\left(t_{2}-s\right)^{\alpha-1} y(s) d s\right.
\end{aligned}
$$


this implies that

$$
\begin{aligned}
\left\|F_{1} y\left(t_{2}\right)-F_{1} y\left(t_{1}\right)\right\|_{2} & \leq \frac{r}{\Gamma(\alpha)}\left[\int_{0}^{t_{2}} \frac{\left(t_{2}-s\right)^{1-\alpha}-\left(t_{1}-s\right)^{1-\alpha}}{\left(t_{2}-s\right)^{1-\alpha}\left(t_{1}-s\right)^{1-\alpha}} d s\right. \\
& \left.+\int_{t_{1}}^{t_{2}}\left(t_{2}-s\right)^{\alpha-1} d s\right] .
\end{aligned}
$$

In a similar way,

$$
\begin{aligned}
\left\|F_{2}(x, y)\left(t_{2}\right)-F_{2}(x, y)\left(t_{1}\right)\right\|_{2} & \left.\leq \frac{L+q r}{\Gamma(1-\alpha)}\left[\int_{0}^{t_{1}} \frac{\left(t_{2}-s\right)^{\alpha}-\left(t_{1}-s\right)^{\alpha}}{\left(t_{2}-s\right)^{\alpha}\left(t_{1}-s\right)^{\alpha}} d s+\int_{t_{1}}^{t_{2}}\left(t_{2}-s\right)^{-\alpha} d s\right\}\right] \\
& +\frac{K+b r}{\Gamma(2-\alpha)}\left[\sqrt{\int_{0}^{t_{1}}\left|\left(t_{2}-s\right)^{1-\alpha}-\left(t_{1}-s\right)^{1-\alpha}\right|^{2} d s}\right. \\
& \left.+\sqrt{\int_{t_{1}}^{t_{2}}\left(t_{2}-s\right)^{2-2 \alpha} d s}\right] .
\end{aligned}
$$

However,

$$
\begin{aligned}
F\left(x\left(t_{2}\right), y\left(t_{2}\right)\right)-F\left(x\left(t_{1}\right), y\left(t_{1}\right)\right) & =\left(F_{1} y\left(t_{2}\right), F_{2}(x, y)\left(t_{2}\right)\right)-\left(F_{1} y\left(t_{1}\right), F_{2}(x, y)\left(t_{1}\right)\right) \\
& =\left(\left(F_{1} y\left(t_{2}\right)-F_{1} y\left(t_{1}\right),\left(F_{2}(x, y)\left(t_{2}\right)-F_{2}(x, y)\left(t_{1}\right)\right)\right.\right.
\end{aligned}
$$

then from (12) and (13), we deduce the equicontinuity of the class $\{F(x, y)(t)\}$ on $Q$.

Consider $\left(x_{n}, y_{n}\right) \in Q$ such that L.i. $m_{n \rightarrow \infty}\left(x_{n}, y_{n}\right)=(x, y)$ w.p.1 where L.i.m denotes the limit in the mean square sense of the continuous second-order process $([23,25,26])$ then by the Arzela-Ascoli Theorem [25], the closure of $F Q$ is a compact subset of $\Lambda$.

Now applying stochastic Lebesgue dominated convergence Theorem [25], we can obtain

$$
\begin{aligned}
\operatorname{Lii.m}_{n \rightarrow \infty} F\left(x_{n}, y_{n}\right)= & \left(\operatorname{Liim}_{n \rightarrow \infty} F_{1} y_{n}, \operatorname{L.i.m}_{n \rightarrow \infty} F_{2}\left(x_{n}, y_{n}\right)\right) \\
= & \left(\operatorname{Liim}_{n \rightarrow \infty}\left\{x_{0}-\int_{0}^{\tau} h_{1}\left(s, I^{\alpha-\beta} y_{n}(s)\right) d W(s)+I^{\alpha} y_{n}(t)\right\},\right. \\
& \text { L.i.m } \left.m_{n \rightarrow \infty}\left\{I^{1-\alpha} g\left(t, x_{n}(\phi(t))\right)+\int_{0}^{t} \frac{(t-s)^{-\alpha}}{\Gamma(2-\alpha)} f\left(s, y_{n}(s)\right) d W(s)\right\}\right) \\
= & \left(F_{1} y, F_{2} x\right)=F(x, y) .
\end{aligned}
$$

This proves that the operator $F: Q \rightarrow Q$ is continuous, and then applying the Schauder Fixed Point Theorem [25], there exists at least one solution $(x, y) \in \Lambda$ of the problem (9) and (10) $x, y \in C\left(I, L_{2}(\Omega)\right)$.

\section{Uniqueness of the Solution}

Consider the assumptions

(B2*) $f: I \times L_{2}(\Omega) \rightarrow L_{2}(\Omega)$ is measurable in $t \in I$ for all $x \in L_{2}(\Omega)$ and satisfies the Lipschitz condition

$$
\|f(t, u(t))-f(t, v(t))\|_{2} \leq b\|u(t)-v(t)\|_{2} .
$$

$\left(\mathrm{B}^{*}\right) h: I \times L_{2}(\Omega) \rightarrow L_{2}(\Omega)$ is measurable in $t \in I \quad$ for all $x \in L_{2}(\Omega)$ and satisfies the Lipschitz condition

$$
\|h(t, u(t))-h(t, v(t))\|_{2} \leq c\|u(t)-v(t)\|_{2} .
$$

$\left(\mathrm{B}^{*}\right) g: I \times L_{2}(\Omega) \rightarrow L_{2}(\Omega)$ is measurable in $t \in I \quad$ for all $x \in L_{2}(\Omega)$ and satisfies the Lipschitz condition

$$
\|g(t, u(t))-g(t, v(t))\|_{2} \leq q\|u(t)-v(t)\|_{2} .
$$


Theorem 2. Let the assumptions $\left(B 2^{*}\right)-\left(B 4^{*}\right)$ and (B5) and (B6) be satisfied, and then the solution of problem (9) and (10) is unique.

Proof. Let $\left(x_{1}, y_{1}\right)$ and $\left(x_{2}, y_{2}\right)$ be two solutions of the problem (9) and (10) on the form

$$
\begin{aligned}
(x(t), y(t))=\left(x_{0}\right. & -\int_{0}^{\tau} h\left(s, I^{\alpha-\beta} y(s)\right) d W(s)+I^{\alpha} y(t), \\
y_{0} & \left.-I^{1-\alpha} g(t, x(\phi(t)))+\int_{0}^{t} \frac{(t-s)^{1-\alpha}}{\Gamma(2-\alpha)} f(s, y(s)) d W(s)\right),
\end{aligned}
$$

then we can obtain

$$
\begin{aligned}
\left\|x_{1}(t)-x_{2}(t)\right\|_{2} & \leq\left\|\int_{0}^{\tau}\left[h\left(s, I^{\alpha-\beta} y_{2}(s)\right)-h\left(s, I^{\alpha-\beta} y_{1}(s)\right)\right] d W(s)\right\|_{2}+\left\|I^{\alpha} y_{1}(t)-I^{\alpha} y_{2}(t)\right\|_{2} \\
& \leq\left[\frac{c T^{\alpha-\beta+\frac{1}{2}}}{\Gamma(1+\alpha-\beta)}+\frac{T^{\alpha}}{\Gamma(1+\alpha)}\left\|y_{1}-y_{2}\right\|_{C} .\right.
\end{aligned}
$$

Similarly, we can obtain

$$
\begin{aligned}
\left\|y_{1}(t)-y_{2}(t)\right\|_{2} & \leq\left\|I^{1-\alpha} g\left(t, x_{1}(\phi(t))\right)-I^{1-\alpha} g\left(t, x_{2}(\phi(t))\right)\right\|_{2} \\
& \left.+\| \int_{0}^{t} \frac{(t-s)^{1-\alpha}}{\Gamma(2-\alpha)}\left[f\left(s, y_{1}(s)\right)-f\left(s, y_{2}(s)\right)\right] d W(s)\right) \|_{2} \\
& \leq \frac{q T^{1-\alpha}}{\Gamma(2-\alpha)}\left\|x_{1}-x_{2}\right\|_{C}+\frac{b T^{\frac{3}{2}-\alpha}}{\Gamma(2-\alpha)}\left\|y_{1}-y_{2}\right\|_{C} .
\end{aligned}
$$

However,

$$
\begin{aligned}
\left\|\left(x_{1}, y_{1}\right)-\left(x_{2}, y_{2}\right)\right\|_{\Lambda} & =\left\|\left(x_{1}-x_{2}\right)\right\|_{C}+\left\|\left(y_{1}-y_{2}\right)\right\|_{C} \\
& \leq \frac{q T^{1-\alpha}}{\Gamma(2-\alpha)}\left\|x_{1}-x_{2}\right\|_{C}+\left[\frac{b T^{\frac{3}{2}-\alpha}}{\Gamma(2-\alpha)}+\frac{c T^{\alpha-\beta+\frac{1}{2}}}{\Gamma(1+\alpha-\beta)}+\frac{T^{\alpha}}{\Gamma(1+\alpha)}\right]\left\|y_{1}-y_{2}\right\|_{C} \\
& \leq\left[\frac{q T^{1-\alpha}}{\Gamma(2-\alpha)}+\frac{b T^{\frac{3}{2}-\alpha}}{\Gamma(2-\alpha)}+\frac{c T^{\alpha-\beta+\frac{1}{2}}}{\Gamma(1+\alpha-\beta)}+\frac{T^{\alpha}}{\Gamma(1+\alpha)}\right]\left[\left\|x_{1}-x_{2}\right\|_{C}+\left\|y_{1}-y_{2}\right\|_{C}\right] \\
& \leq\left[\frac{q T^{1-\alpha}}{\Gamma(2-\alpha)}+\frac{b T^{\frac{3}{2}-\alpha}}{\Gamma(2-\alpha)}+\frac{c T^{\alpha-\beta+\frac{1}{2}}}{\Gamma(1+\alpha-\beta)}+\frac{T^{\alpha}}{\Gamma(1+\alpha)}\right]\left\|\left(x_{1}, y_{1}\right)-\left(x_{2}, y_{2}\right)\right\|_{\Lambda} .
\end{aligned}
$$

This implies that

$$
\left(1-\left[\frac{b T^{\frac{3}{2}-\alpha}}{\Gamma(2-\alpha)}+\frac{q T^{1-\alpha}}{\Gamma(2-\alpha)}+\frac{c T^{\alpha-\beta+\frac{1}{2}}}{\Gamma(1+\alpha-\beta)}+\frac{T^{\alpha}}{\Gamma(1+\alpha)}\right]\right)\left\|\left(x_{1}, y_{1}\right)-\left(x_{2}, y_{2}\right)\right\|_{\Lambda} \leq 0
$$

Then

$$
\left\|\left(x_{1}, y_{1}\right)-\left(x_{2}, y_{2}\right)\right\|_{\Lambda}=0
$$

and $\left(x_{1}, y_{1}\right)=\left(x_{2}, y_{2}\right)$ which proves that the solution of the problem (9) and (10) is unique.

\section{Continuous Dependence}

We shall present the main definitions related to the concepts of continuous dependence of the solution [27].

Definition 2. The solution $(x, y) \in \Lambda$ of the nonlocal problem (9) and (10) is continuously dependent (on the random initial value $x_{0}$ ) if for all $\epsilon>0$, there exists $\delta_{1}>0$ such that $\left\|x_{0}-x_{0}^{*}\right\|_{2} \leq \delta_{1}$ implies that $\left\|x-x^{*}\right\|_{C} \leq \epsilon$.

For this, we have the following theorem. 
Theorem 3. The unique solution of the system (9) and (10) depends continuously on the random data $x_{0}$.

Proof. Let $\left(x^{*}, y^{*}\right)$ be the solution of the system

$$
\begin{aligned}
& x^{*}(t)=x_{0}^{*}-\int_{0}^{\tau} h\left(s, I^{\alpha-\beta} y^{*}(s)\right) d W(s)+I^{\alpha} y^{*}(t) \\
& y^{*}(t)=I^{1-\alpha} g\left(t, x^{*}(\phi(t))\right)+\int_{0}^{t} \frac{(t-s)^{1-\alpha}}{\Gamma(2-\alpha)} f\left(s, y^{*}(s)\right) d W(s),
\end{aligned}
$$

such that $\left\|x_{0}-x_{0}^{*}\right\|_{2}<\delta_{1}$. Then we have

$$
\begin{aligned}
\left\|x(t)-x^{*}(t)\right\|_{2} & \leq\left\|x_{0}-x_{0}^{*}\right\|_{2}+\left\|\int_{0}^{\tau}\left[h\left(s, I^{\alpha-\beta} y(s)\right)-h\left(s, I^{\alpha-\beta} y^{*}(s)\right)\right] d W(s)\right\|_{2}+\left\|I^{\alpha} y(t)-I^{\alpha} y^{*}(t)\right\|_{2} \\
& \leq \delta_{1}+\left[\frac{c T^{\alpha-\beta+\frac{1}{2}}}{\Gamma(1+\alpha-\beta)}+\frac{T^{\alpha}}{\Gamma(1+\alpha)}\right]\left\|y_{1}-y_{2}\right\|_{C}
\end{aligned}
$$

and

$$
\left\|y-y^{*}\right\|_{2} \leq \frac{q T^{1-\alpha}}{\Gamma(2-\alpha)}\left\|x-x^{*}\right\|_{C}+\frac{b T^{\frac{3}{2}-\alpha}}{\Gamma(2-\alpha)}\left\|y-y^{*}\right\|_{C}
$$

and we can obtain that

$$
\begin{aligned}
\left\|(x, y)-\left(x^{*}, y^{*}\right)\right\|_{\Lambda} & =\left\|x-x^{*}\right\|_{C}+\left\|y-y^{*}\right\|_{C} \\
& \leq \delta_{1}+\frac{q T^{1-\alpha}}{\Gamma(2-\alpha)}\left\|x-x^{*}\right\|_{C} \\
& +\left[\frac{b T^{\frac{3}{2}-\alpha}}{\Gamma(2-\alpha)}+\frac{c T^{\alpha-\beta+\frac{1}{2}}}{\Gamma(1+\alpha-\beta)}+\frac{T^{\alpha}}{\Gamma(1+\alpha)}\left\|y-y^{*}\right\|_{C}\right. \\
& \leq \delta_{1}+\left[\frac{q T^{1-\alpha}}{\Gamma(2-\alpha)}+\frac{b T^{\frac{3}{2}-\alpha}}{\Gamma(2-\alpha)}+\frac{c T^{\alpha-\beta+\frac{1}{2}}}{\Gamma(1+\alpha-\beta)}+\frac{T^{\alpha}}{\Gamma(1+\alpha)}\right]\left\|(x, y)-\left(x^{*}, y^{*}\right)\right\|_{\Lambda}
\end{aligned}
$$

which gives our result

$$
\left\|(x, y)-\left(x^{*}, y^{*}\right)\right\|_{\Lambda} \leq \frac{\delta_{1}}{\left(1-\left[\frac{b T^{\frac{3}{2}-\alpha}}{\Gamma(2-\alpha)}+\frac{q T^{1-\alpha}}{\Gamma(2-\alpha)}+\frac{c T^{\alpha-\beta+\frac{1}{2}}}{\Gamma(1+\alpha-\beta)}+\frac{T^{\alpha}}{\Gamma(1+\alpha)}\right]\right)}=\epsilon .
$$

Definition 3. The solution $(x, y) \in \Lambda$ of the nonlocal problem (9) and (10) is continuously dependent (on the random function $h$ ) if for all $\epsilon>0$, there exists $\delta_{2}>0$ such that $\| h^{*}(t, u(t))-$ $h(t, u(t)) \|_{2} \leq \delta_{2}$ implies that $\left\|x-x^{*}\right\|_{C} \leq \epsilon$.

Theorem 4. The unique solution of the system (9) and (10) depends continuously on the random functions $h$.

Proof. Let $\left(x^{*}, y^{*}\right)$ be the solution of the system of stochastic integral Equations (9) and (10) such that

$$
\begin{aligned}
& x^{*}(t)=x_{0}-\int_{0}^{\tau} h^{*}\left(s, I^{\alpha-\beta} y^{*}(s)\right) d W(s)+I^{\alpha} y^{*}(t) \\
& y^{*}(t)=I^{1-\alpha} g\left(t, x^{*}(\phi(t))\right)+\int_{0}^{t} \frac{(t-s)^{1-\alpha}}{\Gamma(2-\alpha)} f\left(s, x^{*}(s)\right) d W(s) .
\end{aligned}
$$

Let $\left\|h^{*}(t, u(t))-h(t, u(t))\right\|_{2} \leq \delta_{2}$ then 


$$
\begin{aligned}
\left\|x(t)-x^{*}(t)\right\|_{2} & \leq\left\|\int_{0}^{\tau}\left[h^{*}\left(s, I^{\alpha-\beta} y^{*}(s)\right)-h\left(s, I^{\alpha-\beta} y(s)\right)\right] d W(s)\right\|_{2}+\left\|I^{\alpha} y(t)-I^{\alpha} y^{*}(t)\right\|_{2} \\
& \leq \delta_{2} \sqrt{T}+\left[\frac{c T^{\alpha-\beta+\frac{1}{2}}}{\Gamma(1+\alpha-\beta)}+\frac{T^{\alpha}}{\Gamma(1+\alpha)}\right]\left\|y-y^{*}\right\|_{C} .
\end{aligned}
$$

Similarly, we can obtain

$$
\left\|y(t)-y^{*}(t)\right\|_{2} \leq \frac{q T^{1-\alpha}}{\Gamma(2-\alpha)}\left\|x-x^{*}\right\|_{C}+\frac{b T^{\frac{3}{2}-\alpha}}{\Gamma(2-\alpha)}\left\|y-y^{*}\right\|_{C}
$$

and

$$
\begin{aligned}
\left\|(x, y)-\left(x^{*}, y^{*}\right)\right\|_{\Lambda} & =\left\|x-x^{*}\right\|_{C}+\left\|y-y^{*}\right\|_{C} \\
& \left.\leq \delta_{2} \sqrt{T}+\frac{b T^{\frac{3}{2}-\alpha}}{\Gamma(2-\alpha)}+\frac{q T^{1-\alpha}}{\Gamma(2-\alpha)}+\frac{c T^{\alpha-\beta+\frac{1}{2}}}{\Gamma(1+\alpha-\beta)}+\frac{T^{\alpha}}{\Gamma(1+\alpha)}\right]\left\|(x, y)-\left(x^{*}, y^{*}\right)\right\|_{\Lambda} .
\end{aligned}
$$

This implies that

$$
\left\|(x, y)-\left(x^{*}, y^{*}\right)\right\|_{\Lambda} \leq \frac{\delta_{2} \sqrt{T}}{1-\left[\frac{b T^{\frac{3}{2}-\alpha}}{\Gamma(2-\alpha)}+\frac{q T^{1-\alpha}}{\Gamma(2-\alpha)}+\frac{c T^{\alpha-\beta+\frac{1}{2}}}{\Gamma(1+\alpha-\beta)}+\frac{T^{\alpha}}{\Gamma(1+\alpha)}\right]}=\epsilon
$$

which completes the proof.

Theorem 5. The unique solution of the system of stochastic integral Equations (9) and (10) depends continuously on the random functions $g$.

Proof. Let $\left(x^{*}, y^{*}\right)$ be the solutions of the system (9) and (10) such that

$$
\begin{aligned}
& x^{*}(t)=x_{0}-\int_{0}^{\tau} h\left(s, I^{\alpha-\beta} y^{*}(s)\right) d W(s)+I^{\alpha} y^{*}(t) \\
& y^{*}(t)=I^{1-\alpha} g^{*}\left(t, x^{*}(\phi(t))\right)+\int_{0}^{t} \frac{(t-s)^{1-\alpha}}{\Gamma(2-\alpha)} f\left(s, y^{*}(s)\right) d W(s) .
\end{aligned}
$$

Let $\left\|g^{*}(t, u(t))-g(t, u(t))\right\|_{2} \leq \delta_{3}$ then

$$
\begin{aligned}
\left\|x(t)-x^{*}(t)\right\|_{2} & \leq\left\|\int_{0}^{\tau}\left[h\left(s, I^{\alpha-\beta} y^{*}(s)\right)-h\left(s, I^{\alpha-\beta} y(s)\right)\right] d W(s)\right\|_{2}+\left\|I^{\alpha} y(t)-I^{\alpha} y^{*}(t)\right\|_{2} \\
& \leq \sqrt{\int_{0}^{\tau}\left[c\left\|I^{\alpha-\beta} y^{*}-I^{\alpha-\beta} y\right\|_{2}\right]^{2} d s}+\frac{T^{\alpha}}{\Gamma(1+\alpha)}\left\|y-y^{*}\right\|_{C} \\
& \leq\left[\frac{c T^{\alpha-\beta+\frac{1}{2}}}{\Gamma(1+\alpha-\beta)}+\frac{T^{\alpha}}{\Gamma(1+\alpha)}\right]\left\|y-y^{*}\right\|_{C}
\end{aligned}
$$


and

$$
\begin{aligned}
\left\|y(t)-y^{*}(t)\right\|_{2} & \leq\left\|I^{1-\alpha} g(t, x(\phi(t)))-I^{1-\alpha} g^{*}\left(t, x^{*}(\phi(t))\right)\right\|_{2} \\
& +\| \int_{0}^{t} \frac{(t-s)^{1-\alpha}}{\Gamma(2-\alpha)}\left[f \left(s, y(s)-f\left(s, y^{*}(s)\right] d W(s) \|_{2}\right.\right. \\
& \leq\left\|I^{1-\alpha} g(t, x(\phi(t)))-I^{1-\alpha} g\left(t, x^{*}(\phi(t))\right)\right\|_{2} \\
& +\left\|I^{1-\alpha} g\left(t, x^{*}(\phi(t))\right)-I^{1-\alpha} g^{*}\left(t, x^{*}(\phi(t))\right)\right\|_{2} \\
& +\sqrt{\int_{0}^{t}\left[\left\|f(s, y(s))-f\left(s, y^{*}(s)\right)\right\|_{2} \frac{(t-s)^{1-\alpha}}{\Gamma(2-\alpha)}\right]^{2} d s} \\
& \leq \frac{q T^{1-\alpha}}{\Gamma(2-\alpha)}\left\|x-x^{*}\right\|_{C}+\frac{\delta_{3} T^{1-\alpha}}{\Gamma(2-\alpha)}+\frac{b T^{\frac{3}{2}-\alpha}}{\Gamma(2-\alpha)}\left\|y-y^{*}\right\|_{C} .
\end{aligned}
$$

Now

$$
\begin{aligned}
\left\|(x, y)-\left(x^{*}, y^{*}\right)\right\|_{\Lambda} & =\left\|x-x^{*}\right\|_{C}+\left\|y-y^{*}\right\|_{C} \\
& \leq \frac{\delta_{3} T^{1-\alpha}}{\Gamma(2-\alpha)}+\frac{q T^{1-\alpha}}{\Gamma(2-\alpha)}\left\|x-x^{*}\right\|_{C} \\
& +\left[\frac{c T^{\alpha-\beta+\frac{1}{2}}}{\Gamma(1+\alpha-\beta)}+\frac{T^{\alpha}}{\Gamma(1+\alpha)}+\frac{b T^{\frac{3}{2}-\alpha}}{\Gamma(2-\alpha)}\right]\left\|y-y^{*}\right\|_{C} \\
& \leq \frac{\delta_{3} T^{1-\alpha}}{\Gamma(2-\alpha)} \\
& +\left[\frac{c T^{\alpha-\beta+\frac{1}{2}}}{\Gamma(1+\alpha-\beta)}+\frac{T^{\alpha}}{\Gamma(1+\alpha)}+\frac{b T^{\frac{3}{2}-\alpha}}{\Gamma(2-\alpha)}+\frac{q T^{1-\alpha}}{\Gamma(2-\alpha)}\right]\left\|(x, y)-\left(x^{*}, y^{*}\right)\right\|_{\Lambda} .
\end{aligned}
$$

This implies that

$$
\left\|(x, y)-\left(x^{*}, y^{*}\right)\right\|_{\Lambda} \leq \frac{\frac{T^{1-\alpha}}{\Gamma(2-\alpha)} \delta_{3}}{1-\left[\frac{c T^{\alpha-\beta+\frac{1}{2}}}{\Gamma(1+\alpha-\beta)}+\frac{T^{\alpha}}{\Gamma(1+\alpha)}+\frac{b T^{\frac{3}{2}-\alpha}}{\Gamma(2-\alpha)}+\frac{q T^{1-\alpha}}{\Gamma(2-\alpha)}\right]}=\epsilon
$$

which completes the proof.

\section{An Example}

Consider the stochastic differential equation

$$
d\left(\frac{d x(t)}{d t}-\frac{x(t) \sin t}{30\left(1+\|x(t)\|_{2}\right)}\right)=\frac{a(t)+D^{\frac{3}{4}} x(t)}{9\left(1+\|x(t)\|_{2}\right)} d W(t), \quad t \in\left(0, \frac{1}{2}\right]
$$

subject to

$$
\begin{aligned}
\left.\frac{d x}{d t}\right|_{t=0} & =0 \\
x_{0} & =\int_{0}^{\tau} \frac{e^{-s} D^{\frac{1}{2}} x(s)}{36+s^{2}} d W(s)
\end{aligned}
$$

where

$$
\begin{aligned}
\|f(t, x(t))\|_{2} & \leq \frac{1}{120}\left[|a(t)|+\|x(t)\|_{2}\right],\|g(t, x(t))\|_{2} \leq \frac{1}{30}\|x(t)\|_{2} \\
\| h(t, x(t) \| & \leq \frac{\|x\|_{2}}{36}
\end{aligned}
$$


Easily, the problem (17) with nonlocal integral conditions (18) satisfies all the assumptions (B1)-(B6) of Theorem 1 with $b=\frac{1}{120}, c=\frac{1}{36}, q=\frac{1}{30}$ then there exists at least one solution to the problem (17) on $\left[0, \frac{1}{2}\right]$.

\section{Conclusions}

Here, we have combined the two senses of derivatives, the stochastic It $\hat{o}$-differential and the arbitrary (fractional) orders derivative, for the second-order stochastic process. The existence of solutions has been proved. The sufficient conditions for the uniqueness of the solution have been given. The continuous dependence of the unique solution has been studied.

Author Contributions: Conceptualization, A.M.A.E.-S. and H.A.F. All authors have read and agreed to the published version of the manuscript.

Funding: This research received no external funding.

Institutional Review Board Statement: Not applicable.

Informed Consent Statement: Not applicable.

Data Availability Statement: Not applicable.

Acknowledgments: The authors would like to thank the referee and the editor for their valuable comments which led to improvement of this work.

Conflicts of Interest: The authors declare no conflict of interest.

\section{References}

1. Adomian, G. Coupled nonlinear stochastic differential equations. J. Math. Anal. Appl. 1983, 92, 427-434. [CrossRef]

2. Elborai, M.M. On some stochastic fractional integro-differential equations. Adv. Dyn. Syst. Appl. 2006, 1, 49-57; ISSN 0973-5321.

3. Soong, T.T. Random Differential Equations in Science and Engineering; Academic Press: New York, NY, USA, 1973.

4. Hafez, F.M.; El-Sayed, A.M.A.; El-Tawil, M.A. On a stochastic fractional calculus. Fractional Calculus Appl. Anal. 2001, 4, 81-90.

5. Hafez, F.M. The Fractional calculus for some stochastic processes. Stoch. Anal. Appl. 2004, 22, 507-523. [CrossRef]

6. El-Sayed, A.M.A.; Fouad, H.A. On a coupled system of random and stochastic nonlinear differential equations with coupled nonlocal random and stochastic nonlinear integral conditions. Mathematics 2021, 9, 2111. [CrossRef]

7. El-Sayed, A.M.A.; Fouad, H.A. On a Coupled System of Stochastic Ito-Differential and the Arbitrary (Fractional) Order Differential Equations with Nonlocal Random and Stochastic Integral Conditions. Mathematics 2021, 9, 2571. [CrossRef]

8. Elsonbaty, A.R.; El-Sayed, A.M.A. Further nonlinear dynamical analysis of simple jerk system with multiple attractors. Nonlinear Dyn. 2017, 87, 1169-1186. [CrossRef]

9. Mou, D.; Fang, J; Fan, Y. Discrete localized excitations for discrete conformable fractional cubic-quintic Ginzburg-Landau model possessing the non-local quintic term. Optik 2021, 244, 167554. [CrossRef]

10. Wu, G.Z.; Yu, L.J.; Wang, Y.Y. Fractional optical solitons of the space-time fractional nonlinear Schrödinger equation. Optik 2020, 207, 164405. [CrossRef]

11. Yu, L.J.; Wua, G.Z.; Wanga, Y.; Chenb, Y.X. Traveling wave solutions constructed by Mittag-Leffler function of a (2+1)-dimensional space-time fractional NLS equation. Results Phys. 2020, 17, 103156. [CrossRef]

12. Ahmad, B.; Ntouyas, S.K.; Alsaedi, A. On a coupled system of fractional differential equations with coupled nonlocal and integral boundary conditions. Chaos Solitons Fractals 2016, 83, 234-224. [CrossRef]

13. Wang, B.H.; Wang, Y.; Dai, C.Q.; Chen, Y.X. Dynamical characteristic of analytical fractional solitons for the space-time fractional Fokas-Lenells equation. Alex. Eng. J. 2020, 59, 4699-4707. [CrossRef]

14. Yin, W.; Cao, J. Nonlocal stochastic differential equations with time-varying delay driven by G-Brownian motion. Math. Meth. Appl. Sci. 2020, 43, 600-612. [CrossRef]

15. Liu, X.; Zhou, Q.; Biswas, A.; Alzahrani, A.K.; Liu, W. The similarities and differences of different plane solitons controlled by (3+ 1)—Dimensional coupled variable coefficient system. J. Adv. Res. 2020, 24, 167-173. [CrossRef]

16. Tsokos, C.P.; Padgett, W.J. Stochastic integral equations in life science and engineering. Int. Stat. Rev. 1973, 412, 15-38. [CrossRef]

17. Wong, E. Stochastic Processes, Informations and Dynamical Systems; McGraw-Hill: New York, NY, USA, 1971.

18. Arqub, O.A.; Shawagfeh, N. Solving optimal control problems of Fredholm constraint optimality via the reproducing kernel Hilbert space method with error estimates and convergence analysis. Math. Methods Appl. Sci. 2021, 44, 7915-7932. [CrossRef]

19. Djennadi, S.; Shawagfeh, N.; Arqub, O.A. A fractional Tikhonov regularization method for an inverse backward and source problems in the time-space fractional diffusion equations. Chaos Solitons Fractals 2021, 150, 111127. [CrossRef]

20. Momani, S.; Arqub, O.A.; Maayah, B. Piecewise optimal fractional reproducing kernel solution and convergence analysis for the Atangana-Baleanu-Caputo model of the Lienard's equation. Fractals 2020, 28, 2040007. [CrossRef] 
21. Momani, S.; Maayah, B.; Arqub, O.A. The reproducing kernel algorithm for numerical solution of Van der Pol damping model in view of the Atangana-Baleanu fractional approach. Fractals 2020, 28, 2040010. [CrossRef]

22. Balasubramaniam1, P.; Tamilalagan1, P. The solvability and optimal controls for impulsive fractional stochastic integro-differential equations via resolvent Operators. J. Optim. Theory Appl. 2017, 174, 139-155. [CrossRef]

23. El-Sayed, A.M.A. On the stochastic fractional calculus operators. J. Fract. Calc. Appl. 2015, 6, 101-109.

24. Krylov, N.V. On Itô's stochastic integral equations. Theory Probab. Its Appl. 1967, 14, 330-336. [CrossRef]

25. Curtain, R.F.; Pritchard, A.J. Functional Analysis in Modern Applied Mathematics; Academic Press: Cambridge, MA, USA, 1977.

26. El-Sayed, A.M.A.; El-Tawil, M.A.; Saif, M.S.M.; Hafez, F.M. The mean square Riemann-Liouville stochastic fractional derivative and stochastic fractional order differential equation. Math. Sci. Res. J. 2005, 9, 142-150.

27. El-Sayed, A.M.A.; Gaafar, F.; El-Gendy, M. Continuous dependence of the solution of Ito stochastic differential equation with nonlocal conditions. Appl. Math. Sci. 2016, 10, 1971-1982. [CrossRef] 